Research Paper

\title{
MicroRNA-365 Inhibits Vascular Smooth Muscle Cell Proliferation through Targeting Cyclin DI
}

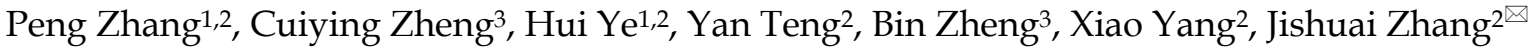 \\ 1. Model Organism Division, E-institutes of Shanghai Universities, Shanghai Jiao Tong University, School of Medicine, Shanghai, 200025, \\ China; \\ 2. State Key Laboratory of Proteomics, Genetic Laboratory of Development and Disease, Institute of Biotechnology, Beijing, 100071, China; \\ 3. Department of Biochemistry and Molecular Biology, Key Laboratory of Neural and Vascular Biology, Ministry of Education, Hebei \\ Medical University, Shijiazhuang, 050017, China.
}

$\square$ Corresponding author: E-mail address: kdliu@zzu.edu.cn

(c) Ivyspring International Publisher. This is an open-access article distributed under the terms of the Creative Commons License (http://creativecommons.org/ licenses/by-nc-nd/3.0/). Reproduction is permitted for personal, noncommercial use, provided that the article is in whole, unmodified, and properly cited.

Received: 2014.02.26; Accepted: 2014.03.26; Published: 2014.05.2I

\begin{abstract}
MicroRNA-365 (miR-365) plays crucial roles in regulating cell proliferation, apoptosis and differentiation in various cell types. However, its function in vascular smooth muscle cells (VSMCs) is largely unknown. In our study, we found miR-365 was highly expressed in adult rat carotid arteries, but was significantly decreased in rat carotid arteries after balloon injury, a process involving neointima formation and VSMC proliferation. In vitro, the miR-365 significantly inhibited cell proliferation of isolated primary rat aortic VSMCs. Furthermore, we identified that cyclin DI was a direct target of miR-365 in VSMCs. The miR-365 suppressed cyclin DI expression on both mRNA and protein level. Luciferase reporter assay demonstrated that miR-365 inhibited cyclin DI through targeting its 3'UTR. Importantly, cyclin DI overexpression rescued the inhibitory effect of miR-365 on VSMCs proliferation. Taken together, by our studies, we identified a new MicroRNA, miR-365, involving in the pathological process of vascular injury, which inhibits VSMC proliferation through targeting cyclinDI.
\end{abstract}

Key words: miR-365; vascular smooth muscle cells; proliferation; neointima formation; cyclin D1.

\section{Introduction}

Vascular smooth muscle cells (VSMCs) are the main cell type of vascular wall and play an important role in vascular development and disease. VSMCs have high rate of proliferation and migration during early embryonic stages of vasculogenesis, while in adult blood vessels VSMCs become quiescent and proliferate at a very low rate [1]. Unlike skeletal and cardiac muscle cells which are terminally differentiated cells, VSMCs retain remarkable plasticity and can undergo phenotypic switching. In various forms of vascular diseases such as atherosclerosis, restenosis, vein bypass graft failure, and transplant vasculopathy, VSMCs switch from contracting phenotype to secreting phenotype, proliferate, and migrate into intima, contributing to neointima formation [2].

MicroRNAs (miRNAs) are endogenous, sin- gle-stranded RNA of 22 nucleotides and constitute a novel class of gene regulators [3, 4]. Increasing evidence indicates that MicroRNAs play critical roles in various VSMC functions and vascular diseases $[5,6]$. In particular, miR-1, miR-31, miR-133, miR-143/145, miR-146a and miR-638 are involved in neointima formation after vascular injury by regulating VSMC proliferation, migration and phenotypic switching [7-13]. MicroRNA-365 (miR-365) is reported to regulate cell proliferation, apoptosis or differentiation in colon cancer cell lines [14], lung cancer cell lines [15], HaCaT cells [16], chondrocytes [17] and endothelial cells [18]. Our previous work showed that miR-365 inhibited gastric cancer cells proliferation, and participated in gastric cancer formation [19]. However, the function of miR-365 in VSMCs and neointima 
formation is still unknown.

In the present work, we demonstrated that miR-365 expression was significantly decreased in neointima formation model of rat carotid arteries in vivo, and miR-365 inhibited cell proliferation through targeting cyclin D1 in primary rat VSMCs in vitro. Our findings suggest that miR-365 probably is a novel important modulator for neointima formation.

\section{Materials and methods}

\section{Cell culture and transfection}

Primary vascular smooth muscle cells were isolated from the thoracic aortas of male Sprague-Dawley rats as previously described [9]. The VSMCs were cultured in Dulbecco's modified Eagle's medium (DMEM)/F12 medium containing 10\% fetal bovine serum (FBS, SV30087.02, Hyclone) with 1\% penicillin-streptomycin, and maintained at $37^{\circ} \mathrm{C}$ in a humidified atmosphere containing $5 \% \mathrm{CO} 2$. The cells used in the experiments were from passage 3 to 5 . Cells were individually plated on tissue culture plates or dishes $24 \mathrm{~h}$ before transfection. Transient transfection of miRNA mimics and control oligonucleotides (Genepharm) at a final concentration of $50 \mathrm{nM}$ was accomplished with polyplus reagent (jetPRIME®). The cyclin D1 expression vector was constructed by inserting human cyclin D1 cDNA into the pCMV-HA vector ( $\mathrm{pCMV}-\mathrm{HA}$-cyclinD1).

\section{Proliferation assays}

Cell count: $5 \times 10^{4}$ cells were seeded into 24 -well plate in triplicate, and then transfected with miRNAs as described above [19]. After 6h transfection, the medium was changed into serum-free DMEM/F12 for starvation. 24 hours later, normal serum medium (10\% FBS) was added (0h), and the cell number was counted at $24 \mathrm{~h}, 48 \mathrm{~h}$, or $72 \mathrm{~h}$ under microscope using a hemocytometer.

BrdU incorporation assay: BrdU incorporation assay was performed and analyzed as described previously [10]. Briefly, cells grown on glass coverslips were labeled with $10 \mu \mathrm{M}$ BrdU every eight hours. 24 hours later, the cells were fixed in $4 \%$ paraformaldehyde (PFA) for 30 minutes, and permeabilized in $0.5 \%$ Triton X-100 in PBS (pH 7.4) for 10 minutes. The fixed cells were incubated with $4 \mathrm{~N}$ hydrochloric acid for 30 minutes at $37^{\circ}$, and were pre-blocked with goat serum for 30 minutes, and then incubated with rat anti-BrdU antibody (ab6326; 1:100; abcam) overnight at $4^{\circ} \mathrm{C}$. The sections were washed in PBS and incubated with goat anti-rat IgG/FITC for $1 \mathrm{~h}$ at room temperature (ZF-0315; 1:200; ZSGB-Bio, Beijing, China). The nuclei were labeled with 4', 6-diamidino-2-phenylindole (DAPI). The BrdU incorporation rate was expressed as the percentage of BrdU-positive cells to total cell number per field.

\section{Luciferase activity assay}

The luciferase reporter plasmid containing human cyclin D1 3'-UTR sequence or mutant putative miR-365 seed sequence was generated as previously described [19]. The reporter plasmids, together with miR-365 or miRNA negative control, were transfected into cells using polyplus reagent (jetPRIME®), and luciferase activity was measured with the Luciferase assay kit (Promega).

\section{Western blot}

Cells were homogenized in lysis buffer supplemented with protease inhibitor cocktail (Roche). Proteins $(25 \mathrm{ug})$ were electrophoresed on SDS-PAGE and transferred onto PVDF membranes. Immunoblotting was done using rabbit anti-cyclin D1 (2261-S; 1:1000; epitomics) and mouse anti-GAPDH (sc-25778; 1:3000; ZSGB-Bio) antibodies.

\section{Determination of RNA levels by Real-time PCR}

miRNAs were extracted from rat adult aortic VSMCs, aorta, carotid artery, heart, liver, spleen, lung, kidney, stomach and intestine by Trizol. The expression levels of mature miR-365 and U6 were measured using the Applied Biosystems TaqMan MicroRNA Assays system (7500 Fast; Applied Biosystems $\left.{ }^{\circledR}\right)$. U6 expression was used to normalize the expression of miR-365. The $2^{-\Delta C t}$ method was used to analyze Real-time PCR data.

\section{Balloon injury model}

Carotid artery balloon injury was induced in male Sprague-Dawley rats (300 g) as previously described [9]. In brief, animals were anesthetized with urethane $(600 \mathrm{mg} / \mathrm{kg})$ intraperitoneally. The left common carotid artery was exposed through a midline cervical incision. The catheter was advanced from just under the proximal edge of the omohyoid muscle to the carotid bifurcation three times with a $2 \mathrm{~F}(60 \mathrm{~cm})$ Fogarty catheter (Baxter, McGaw Park, IL). To attain a constant degree of vessel wall injury for each of the animals, we kept the diameter of the balloon and the resistance during withdrawal constant for each of the animals. All procedures were performed by a single operator. Five rats were used in each group. All animal experiments were approved by the Animal Experiment Committee of the Institute of Biotechnology.

\section{Histological analysis and immunohistochem- istry}

At 14 days after surgery, left carotid arteries were dissected and fixed with $4 \%$ PFA overnight. The 
arteries were subsequently paraffin-embedded, and serial $5-\mu \mathrm{m}$ sections were cut. For morphometric analysis, sections were stained with hematoxylin and eosin (H\&E) after deparaffinisation and rehydration. Immunohistochemistry staining of cyclin D1 was done using rabbit anti-cyclin D1 (2261-S; 1:200; epitomics) and goat anti-rabbit IHC kit (SP-9001; 1:200; ZSGB-Bio)

\section{Statistical analysis}

All values were expressed as mean \pm SD. Statistical analysis was performed by 2-tailed Student's $t$ test between two groups. The differences among three groups were determined using one-way analysis of variance (ANOVA) followed by Bonferroni's post-hoc tests. Results were considered statistically significant at $P<0.05$.

\section{Results}

miR-365 is expressed in rat carotid arteries, and is significantly decreased after balloon injury

When we detected miR-365 expression pattern in various tissues of rat by real-time PCR, we observed that, except stomach, miR-365 was significantly more expressed in aorta and carotid mainly composed of vascular smooth muscle cells, compared to other tissues (heat, liver, lung etc) (Fig. 1A). To evaluate whether miR-365 was involved in neointima formation, we subjected rats to carotid balloon injury, a well-established in vivo experimental model to study neointima formation [20]. At 14 days after surgery, the injured carotid shows obviously narrow vascular cavity, with thicker intima and media compared to no injury control (Fig. 1B). Real-time PCR analysis revealed that miR-365 expression was significantly down-regulated in the injured carotid arteries compared to control group (Fig. $1 \mathrm{C}, \mathrm{n}=5, P<0.01$ ). These results suggested that miR-365 may play a role in neointima formation.

\section{miR-365 inhibits VSMC proliferation}

The increased VSMC proliferation is contributed to the pathogenesis of neointima formation [21]. In our study, we evaluated the function of miR-365 in VSMC proliferation. The miR-365 was over-expressed using chemically synthesized mimic in primary rat aortic vascular smooth muscle cells. The Real-time PCR results showed that miR-365 expression was significantly increased by 51.3 folds after transfection (Fig. 2A). Next, we counted VSMCs cell number at different time points after miR-365 mimic transfection. At 48 hours post-transfection, the cell number was decreased by $40 \%$ in the
miR-365-mimic-transfected cells compared with negative control (NC) cells $(P<0.01)$, and this decrease was pronounced by $60 \%$ at 72 hours $(P<0.01)$ (Fig. 2B). Furthermore, we detected whether the inhibitory effect of miR-365 on VSMC proliferation was due to cell division through BrdU incorporation. We observed that the percentage of BrdU-positive cells was significantly decreased by $58 \%(P<0.01)$ (Fig. 2C). These results showed that miR-365 significantly inhibits VSMC proliferation.

\section{Cyclin DI is a direct target of miR-365}

To explore the mechanism by which miR-365 inhibited VSMC proliferation, we attempted to identify miR-365 targets in VSMCs. Our previous results showed that cyclin D1 was a direct target of miR-365 in gastric cancer cells [19]. Herein, we firstly evaluated whether miR-365 could suppress cyclin D1 expression in primary rat VSMCs. The mRNA level of cyclin D1 was significantly decreased upon miR-365 transfection (Fig. 3A). Accordingly, the protein level of cyclin D1 was also dramatically reduced after miR-365 overexpression (Fig. 3B). Moreover, we observed that cyclin D1 expression was obviously increased in the injured carotid arteries compared with uninjured group (Fig. 3C, n = 5). These findings showed that the expression of miR-365 is inversely related to the cyclin D1 expression. To further confirm that cyclin D1 was a direct target of miR-365 in VSMCs, we performed the luciferase reporter assay. The results showed that miR-365 transfection significantly inhibited the luciferase activity of wild type cyclin D1 3' UTR, but not the mutant cyclinD1 3' UTR with the mutant biding site of miR-365 (Fig. 3D). These results demonstrated that cyclin D1 is a direct target of miR-365 in vascular smooth muscle cells.

\section{Overexpression of cyclin DI can rescue miR-365's effect on VSMCs proliferation}

To evaluate whether decreased cyclin D1 mediate the inhibitory effect of miR-365 on VSMC proliferation, we transfected cyclin D1 plasmid without 3' UTR in primary VSMCs. The western blot results showed that the expression of cyclin D1 was increased after transfection (Fig. 4A). When VSMCs were simultaneously transfected with cyclin D1 plasmid and miR-365 mimic, the results of cell count demonstrated that cyclin D1 overexpression rescued miR-365 induced growth inhibition (Fig. 4B, $P<0.01$ ). In addition, BrdU incorporation results confirmed that cyclin D1 overexpression blocked the inhibitory effect of miR-365 on VSMC proliferation (Fig. 4C, $P<0.05$ ). Together, these results implied that miR-365 inhibits VSMC proliferation through decreasing cyclin D1. 
A

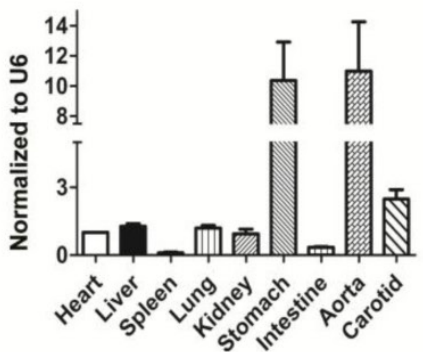

B

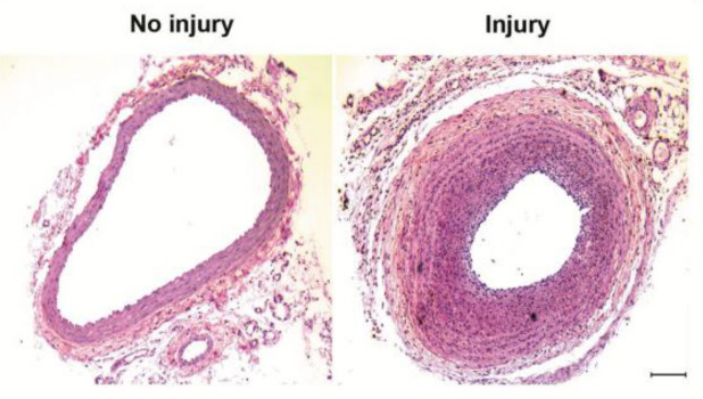

C

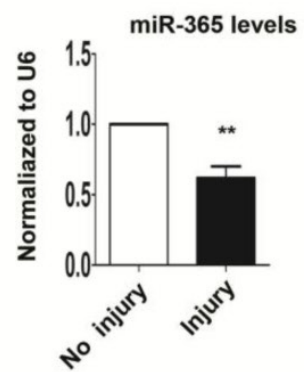

Figure I. miR-365 expression is significantly decreased in neointima formation of rat carotid arteries. (A) Quantitative real-time PCR analyses of miR-365 expression level in various tissues: heart, liver, spleen, lung, kidney, stomach, intestine, aorta and carotid. The expression level of heart was set as I. (B) Micrographs of arterial cross-sections with H \& E staining without injury (No Injury) or at I4 days after injury (Injury) ( $\mathrm{n}=5$ ). Scar bar: $100 \mu \mathrm{m}$ (C) Control and injured arterial segments were harvested for extraction of MicroRNA, followed by measuring the expression of miR-365 using real-time PCR. The expression level of miR-365 was normalized to U6. $* * P<0.01$.

A



B

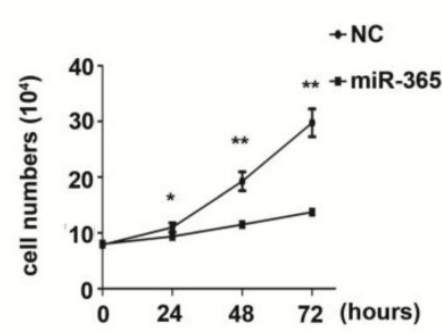

C

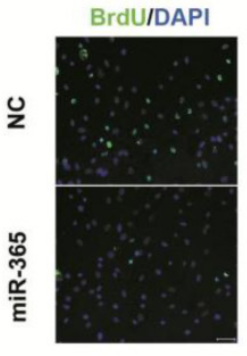

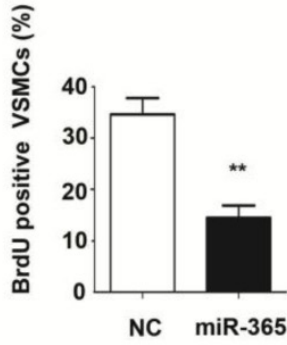

Figure 2. miR-365 inhibits vascular smooth muscle cell proliferation. (A) Quantitative real-time PCR analyses of miR-365 expression after transfection with NC or miR-365 mimic. (B) The effect of miR-365 overexpression on vascular smooth muscle cell proliferation in vitro as measured by cell counts at $0,24,48,72$ hours after transfection with NC or miR-365 mimic. $* P<0.05$, $* * P<0.01$. (C) BrdU incorporation experiment (BrdU, green; DAPI, blue) used as an independent manner to detect VSMCs proliferation at 24 hours after transfection with NC or miR-365 mimic. **p $<0.01$. Scar bar: $50 \mu \mathrm{m}$.

A

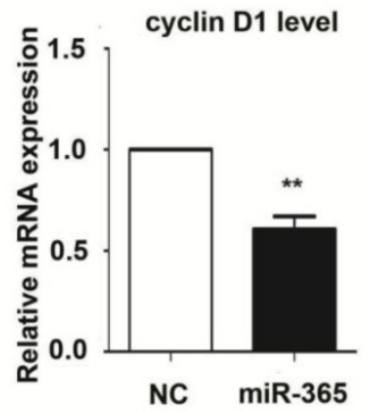

C

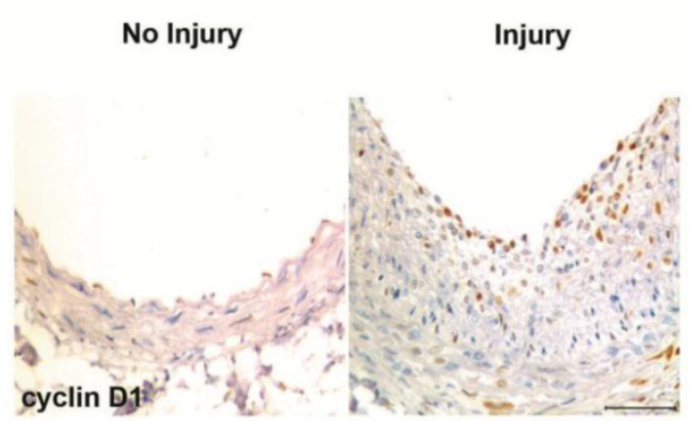

B


Figure 3. cyclin DI is a direct target of miR-365 in vascular smooth muscle cells. (A) Quantitative real-time PCR analyses of cyclin DI expression in VSMCs after transfection with NC or miR-365 mimic. ${ }^{* * P}<0.01$. (B) Left panel: representative western blot of cyclin DI in VSMCs after transfection with NC or miR-365 mimic. Right panel: quantification of cyclin DI via western blot. **P < 0.01 . (C) Representative immunohistochemistry staining of cyclin DI in carotid arteries with or without injury $(n=5)$. Scar bar: $50 \mu \mathrm{m}$. (D) Luciferase activities were measured in VSMCs cotransfected with the reporter constructs containing wild type (WT) or mutant (Mut) 3'UTR of cyclin DI, and NC or miR-365 mimic. **P $<0.0$ I. 


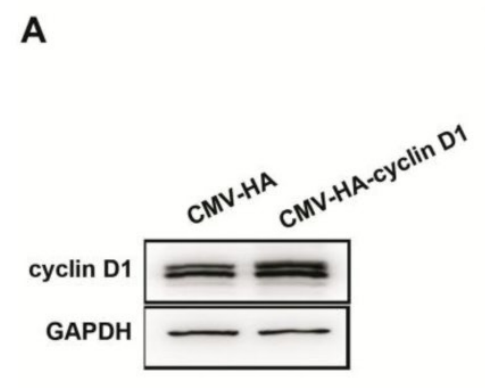

B

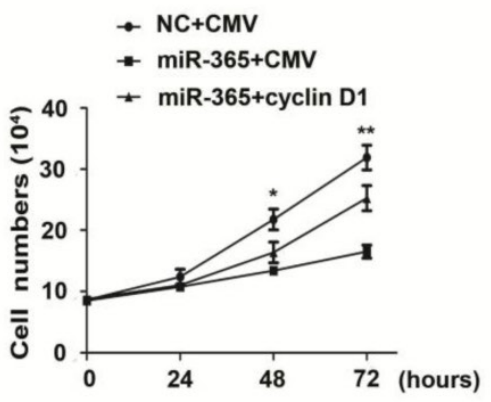

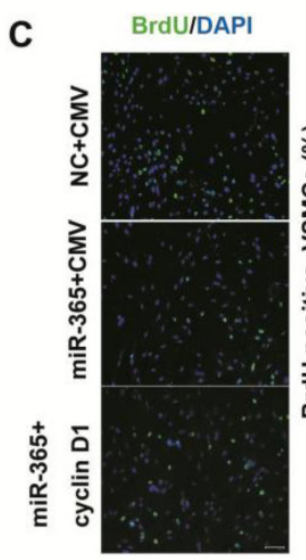

Figure 4. miR-365 inhibits VSMC proliferation by targeting cyclin DI. (A) Representative western blot of cyclin DI expression in VSMCs after transfection with CMV-HA or CMV-HA-cyclin DI overexpression constructs. (B) Cyclin DI overexpression blocked the inhibitory effect of miR-365 on VSMC proliferation as measured by cell count at $0,24,48,72$ hours after transfection with NC plus CMV-HA, miR-365 plus CMV-HA or miR-365 plus CMV-HA-cyclin DI. *P < 0.05, **P < 0.01 for miR-365 plus CMV-HA-cyclin DI vs. miR-365 plus CMV-HA (one way ANOVA and post-hoc tests). (C) Left panel: representative immunofluorescent staining of BrdU (green) and DAPI (blue) showing the VSMC proliferation after transfection with NC plus CMV-HA, miR-365 plus CMV-HA or miR-365 plus CMV-HA-cyclin DI. Scar bar: $50 \mu \mathrm{m}$. Right panel: quantification of BrdU incorporation in VSMCs transfected with NC plus CMV-HA, miR-365 plus CMV-HA or miR-365 plus CMV-HA-cyclin DI. **P $<0.01$ for miR-365 plus CMV-HA vs. NC plus CMV-HA, \#P $<0.05$ for miR-365 plus CMV-HA-cyclin DI vs. miR-365 plus CMV-HA (one way ANOVA and post-hoc tests).

\section{Discussion}

In the present study, we found that miR-365 had a high expression in vascular smooth muscle tissues such as carotid, and was dramatically decreased in carotid arteries after balloon injury. Next, we demonstrated that miR-365 significantly inhibited cell proliferation in primary rat VSMCs. Moreover, we revealed that cyclin D1 was a direct target of miR-365, and cyclin D1 overexpression blocked the inhibitory effect of miR-365 on VSMC proliferation.

For the first time, we identified the function of miR-365 in VSMCs. It has been reported that miR-365 regulates cell proliferation, apoptosis and differentiation in various cell types [14-17]. However, its function in VSMCs is still unknown. In this study, we demonstrated that miR-365 overexpression significantly inhibited VSMC proliferation. Previous works show that miR-365 inhibits cell proliferation in lung cancer [15], gastric cancer [19] while it promotes cell proliferation in cutaneous squamous cell carcinoma [16] and chondrocytes [17], suggesting its function in regulation of cell proliferation is dependent on cell type. Our results showed that miR-365 had an inhibitory effect on cell proliferation in VSMCs.

The VSMC proliferation plays an important role in carotid neointima formation after injury [2]. In response to injury, media VSMCs are activated and migrate to the intima, where they proliferate and subsequently produce abundant extracellular matrix (ECM) to form the neointima [21]. More recently, increasing evidences showed that MicroRNAs participate in VSMC proliferation and neointima formation. The miR-146a, miR-31, miR-221 and miR-222 promoted VSMC proliferation, and inhibition of miR-146,
miR-221 and miR-222 by adenovirus significantly decreased neointima formation in response to balloon injury [8, 9, 22], while miR-133 and miR-1 inhibited VSMC proliferation, and Adeno-miR-133 significantly reduced neointimal hyperplasia after balloon injury $[10,23]$. Our work demonstrated that miR-365 significantly inhibited VSMC proliferation in vitro. In addition, miR-365 expression was significantly decreased in carotid arteries in response to injury in vivo, suggesting miR-365 may be a novel important MicroRNA involved in neointima formation.

Our further study identified cyclin D1 as a direct target of miR-365 in primary VSMCs. To evaluate whether the decreased cyclinD1 mediates the inhibitory effect of miR-365 on VSMCs proliferation, we over-expressed cyclinD1 in primary rat aortic smooth muscle cells, and found that cyclin D1 overexpression partially rescue the inhibition of VSMCs proliferation after miR-365 transfection. The MicroRNA is known to have hundreds of targets to mediate its function [3]. In this study, although we provide evidence to show cyclin D1 is the target of miR-365 in VSMCs, we could not rule out that the other targets mediate the inhibitory effect of miR-365 on VSMC proliferation.

In summary, we demonstrate that miR-365 inhibits VSMC proliferation through targeting cyclin D1. The down-regulation of miR-365 in injured carotid arteries suggests that miR-365 may participate in the neointima formation, and can be used as a potential therapeutic target in preventing vascular proliferation diseases.

\section{Abbreviations}

VSMCs: vascular smooth muscle cells; MicroRNAs: miRNAs. 


\section{Acknowledgements}

This study was funded by the National Natural Science Foundation of China (NO. 81270165), and State Key Laboratory of Proteomics Grant (No. SKLP-K201102).

\section{Competing Interests}

The authors have declared that no competing interest exists.

\section{References}

1. Owens GK, Kumar MS, Wamhoff BR. Molecular regulation of vascular smooth muscle cell differentiation in development and disease. Physiol Rev. 2004; 84: 767-801.

2. Gibbons GH, Dzau VJ. The emerging concept of vascular remodeling. N Engl J Med. 1994; 330: 1431-8

3. Farh KK, Grimson A, Jan C, Lewis BP, Johnston WK, Lim LP, et al. The widespread impact of mammalian MicroRNAs on mRNA repression and evolution. Science. 2005; 310: 1817-21.

4. Ambros V. MicroRNA pathways in flies and worms: growth, death, fat, stress, and timing. Cell. 2003; 113: 673-6.

5. McDonald RA, Hata A, MacLean MR, Morrell NW, Baker AH. MicroRNA and vascular remodelling in acute vascular injury and pulmonary vascular remodelling. Cardiovasc Res. 2011; 93: 594-604.

6. Urbich C, Kuehbacher A, Dimmeler S. Role of microRNAs in vascular diseases, inflammation, and angiogenesis. Cardiovasc Res. 2008; 79: 581-8.

7. Xie C, Huang H, Sun X, Guo Y, Hamblin M, Ritchie RP, et al. MicroRNA-1 regulates smooth muscle cell differentiation by repressing Kruppel-like factor 4. Stem Cells Dev. 2010; 20: 205-10.

8. Liu X, Cheng Y, Chen X, Yang J, Xu L, Zhang C. MicroRNA-31 regulated by the extracellular regulated kinase is involved in vascular smooth muscle cell growth via large tumor suppressor homolog 2. J Biol Chem. 2011; 286: 42371-80.

9. Sun SG, Zheng B, Han M, Fang XM, Li HX, Miao SB, et al. miR-146a and Kruppel-like factor 4 form a feedback loop to participate in vascular smooth muscle cell proliferation. EMBO Rep. 2010; 12: 56-62.

10. Torella D, Iaconetti C, Catalucci D, Ellison GM, Leone A, Waring CD, et al. MicroRNA-133 controls vascular smooth muscle cell phenotypic switch in vitro and vascular remodeling in vivo. Circ Res. 2011; 109: 880-93.

11. Cheng Y, Liu X, Yang J, Lin Y, Xu DZ, Lu Q, et al. MicroRNA-145, a novel smooth muscle cell phenotypic marker and modulator, controls vascular neointimal lesion formation. Circ Res. 2009; 105: 158-66.

12. Davis-Dusenbery BN, Chan MC, Reno KE, Weisman AS, Layne MD, Lagna G, et al. down-regulation of Kruppel-like factor-4 (KLF4) by microRNA-143/145 is critical for modulation of vascular smooth muscle cell phenotype by transforming growth factor-beta and bone morphogenetic protein 4 . J Biol Chem. 2011; 286: 28097-110.

13. Pannu H, Fadulu VT, Chang J, Lafont A, Hasham SN, Sparks E, et al. Mutations in transforming growth factor-beta receptor type II cause familial thoracic aortic aneurysms and dissections. Circulation. 2005; 112: 513-20.

14. Nie J, Liu L, Zheng W, Chen L, Wu X, Xu Y, et al. microRNA-365, down-regulated in colon cancer, inhibits cell cycle progression and promotes apoptosis of colon cancer cells by probably targeting Cyclin D1 and Bcl-2. Carcinogenesis. 2012; 33: 220-5.

15. Kang SM, Lee HJ, Cho JY. MicroRNA-365 regulates NKX2-1, a key mediator of lung cancer. Cancer Lett. 2013; 335: 487-94.

16. Zhou M, Liu W, Ma S, Cao H, Peng X, Guo L, et al. A novel onco-miR-365 induces cutaneous squamous cell carcinoma. Carcinogenesis. 2013; 34: 1653-9.

17. Guan YJ, Yang X, Wei L, Chen Q. MiR-365: a mechanosensitive microRNA stimulates chondrocyte differentiation through targeting histone deacetylase 4. FASEB J. 2011; 25: 4457-66.

18. Qin B, Xiao B, Liang D, Xia J, Li Y, Yang H. MicroRNAs expression in ox-LDL treated HUVECs: MiR-365 modulates apoptosis and Bcl-2 expression. Biochem Biophys Res Commun. 2011; 410: 127-33.

19. Guo SL, Ye H, Teng Y, Wang YL, Yang G, Li XB, et al. Akt-p53-miR-365-cyclin D1/cdc25A axis contributes to gastric tumorigenesis induced by PTEN deficiency. Nat Commun. 2013; 4: 2544.

20. Kumar A, Lindner V. Remodeling with neointima formation in the mouse carotid artery after cessation of blood flow. Arterioscler Thromb Vasc Biol. 1997; 17: 2238-44.

21. ER OB, Ma X, Simard T, Pourdjabbar A, Hibbert B. Pathogenesis of neointima formation following vascular injury. Cardiovasc Hematol Disord Drug Targets. 2011; 11: 30-9.

22. Liu X, Cheng Y, Zhang S, Lin Y, Yang J, Zhang C. A necessary role of miR-221 and miR-222 in vascular smooth muscle cell proliferation and neointimal hyperplasia. Circ Res. 2009; 104: 476-87.
23. Chen J, Yin $\mathrm{H}$, Jiang $\mathrm{Y}$, Radhakrishnan SK, Huang ZP, Li J, et al. Induction of microRNA-1 by myocardin in smooth muscle cells inhibits cell proliferation. Arterioscler Thromb Vasc Biol. 2010; 31: 368-75. 